\title{
The thick disk rotation-metallicity correlation as a fossil of an "inverse chemical gradient" in the early Galaxy
}

\author{
A. Curir ${ }^{1}$, M. G. Lattanzi ${ }^{1}$, A. Spagna ${ }^{1}$, F. Matteucci ${ }^{2}$, G. Murante ${ }^{1}$, P. Re Fiorentin ${ }^{1}$, and E. Spitoni ${ }^{3}$ \\ 1 INAF - Osservatorio Astronomico di Torino, via Osservatorio 20, 10025 Pino Torinese, Italy \\ e-mail: curir@oato.inaf.it \\ 2 Department of Mathematics, Physics and Natural Sciences, University of Trieste, 34127, Trieste, Italy \\ ${ }^{3}$ Department of Mathematics, University of Evora, Portugal
}

Received 1 December 2011 / Accepted 20 July 2012

\begin{abstract}
Context. The thick disk rotation-metallicity correlation, $\partial V_{\phi} / \partial[\mathrm{Fe} / \mathrm{H}]=40 \div 50 \mathrm{~km} \mathrm{~s}^{-1} \mathrm{dex}^{-1}$ represents an important signature of the formation processes of the galactic disk.

Aims. We use nondissipative numerical simulations to follow the evolution of a Milky Way (MW)-like disk to verify if secular dynamical processes can account for this correlation in the old thick disk stellar population.

Methods. We followed the evolution of an ancient disk population represented by 10 million particles whose chemical abundances were assigned by assuming a cosmologically plausible radial metallicity gradient with lower metallicity in the inner regions, as expected for the 10-Gyr-old MW. The two cases of a disk with and without a bar were simulated to compare the evolution of their kinematics and radial chemical properties.

Results. Migration processes act in both cases and appear to be enhanced in the presence of a central bar. Essentially, inner disk stars move towards the outer regions and populate layers located at higher $|z|$. In the case of an evolved barred disk, a rotation-metallicity correlation appears, which well resembles the behaviour observed in our Galaxy at a galactocentric distance between $8 \mathrm{kpc}$ and $10 \mathrm{kpc}$. In particular, we measure a correlation of $\partial V_{\phi} / \partial[\mathrm{Fe} / \mathrm{H}] \simeq 60 \mathrm{~km} \mathrm{~s}^{-1} \mathrm{dex}^{-1}$ for particles at $1.5 \mathrm{kpc}<|z|<2.0 \mathrm{kpc}$ that persists up to 6 Gyr.

Conclusions. Our pure $N$-body models can account for the $V_{\phi}$ vs. $[\mathrm{Fe} / \mathrm{H}]$ correlation observed in the thick disk of our Galaxy, suggesting that processes internal to the disk such as heating and radial migration play a role in the formation of this old stellar component. In this scenario, the positive rotation-metallicity correlation of the old thick disk population would represent the relic signature of an ancient inverse chemical (radial) gradient in the inner Galaxy, which resulted from accretion of primordial gas.
\end{abstract}

Key words. Galaxy: evolution - Galaxy: disk - Galaxy: kinematics and dynamics - Galaxy: abundances - Galaxy: structure methods: numerical

\section{Introduction}

Two distinct disk populations are observed in the solar neighbourhood in terms of kinematics, average metallicities, and stellar ages. They belong to a thin disk, younger than 7-8 Gyr, and to a thick disk, older than 8-9 Gyr (e.g. Haywood 2008, and references therein).

We now have several different scenarios for the origin of the thick disk: kinematical heating of a pre-existing old disk via minor mergers (Quinn et al. 1993; Robin et al. 1996; Villalobos \& Helmi 2008; Qu et al. 2011), accretion of satellites that have deposited their stellar debris in planar configuration (Abadi et al. 2003), gas accretion at high redshift and stars formed in situ (Brook et al. 2005), wet merger (Martel et al. 2011), clumpy disks (Bournaud \& Elmegreen 2009), and radial migration (Schönrich \& Binney 2009a,b; Loebman et al. 2011). The interested reader can consult the work by Lee et al. (2011) for a detailed overview of the possible origins of the thick disk.

Chemical properties of stars provide important clues to disentangle the puzzle of the Galaxy formation. By measuring abundance ratios of stars in different parts of the Galaxy, one

* Figures 3-6 and 8 are available in electronic form at http://www. aanda.org can infer how fast metal enrichment proceeded and the timescale over which the different regions were formed. The distribution of the chemical elements in our Galaxy can be considered as a "fossil record" of its evolutionary history. Therefore crucial information on the mechanism that dominated the formation of the thick disk is encoded in the chemical properties of its stars. By comparing the chemical properties of bulge, thick and thin disk stars, and by correlating them with other kinematical data, one can identify the processes that play a dominant role in the formation of the different Galactic components.

On this matter, an intriguing correlation between thick disk rotation and metallicity was found by Spagna et al. (2010). Subsequently, this correlation was confirmed by Lee et al. (2011) using similar data and, very recently, by Kordopatis et al. (2011) using fully independent spectroscopic data. The Spagna et al. correlation is radically different from what is observed for the thin disk stars (Haywood 2008), and from predictions made by the chemodynamical models of Schönrich \& Binney (2009a) and of Loebman et al. (2011) for young stars.

In the context of stellar migration models, the negative rotation-metallicity correlation shown by the thin disk stars in the solar neighbourhood derives from the "immigration" of slower rotating stars that come from the inner disk regions and 
are characterised by stellar populations with higher chemical abundances.

Conversely, the positive correlation shown by the $V_{\phi}$ vs. $[\mathrm{Fe} / \mathrm{H}]$ distribution of thick disk stars observed in situ above $1 \mathrm{kpc}$ from the plane could be explained with a population of much older stars that come from the inner regions of the Galaxy and have formed during the early disk star formation phases from molecular clouds with relatively low chemical abundance and higher $\alpha$-enrichment. Note that relationships between thick disks and bulges in external galaxies have been known for quite some time (Freeman 1987).

Although this substantially positive $V_{\phi}$ vs. $[\mathrm{Fe} / \mathrm{H}]$ correlation has not yet received much theoretical attention, a weak positive correlation is found in the simulations carried out by Loebman et al. (2011), while the statistical model developed by Schönrich $\&$ Binney (2009a) indicates only a mild trend $\left(10 \mathrm{~km} \mathrm{~s}^{-1} \mathrm{dex}^{-1}\right)$ at $z=0 \mathrm{kpc}$, which decreases with height and disappears for $|z|>1 \mathrm{kpc}$ (Schönrich 2009, priv. comm.).

In this paper we show that if we assume the early radial chemical gradient derived from the inside-out formation and chemical evolution model of the Galactic disk as originally suggested by Matteucci \& Francois (1989) and Chiappini et al. (2001), a positive correlation between rotation velocity and metallicity can be established as a result of radial migration and heating processes of stars from the inner region of the disk. These processes are investigated by evolving a stellar disk with $\mathrm{N}$-body simulations and thus following how the chemical properties assigned to the initial configuration are redistributed.

Our model cannot be viewed as a complete galaxy evolution model, because we neglected the gradual formation and growth of the stellar disk. However, our approach allows us to analyse the dynamical evolution of the non dissipative component, and, therefore, to separate the chemical evolution from dynamics, i.e. leaving any redistribution of chemical gradients to stellar motion.

\section{Observed rotation-metallicity correlation}

The observed rotation-metallicity correlation is based on a new kinematic catalogue derived by assembling the astrometric parameters extracted from the database used for the construction of the Second Guide Star Catalog (GSC-II; Lasker et al. 2008) with spectro-photometric data from the Seventh Data Release of the Sloan Digital Sky Survey (SDSS DR7; see e.g. Abazajian et al. 2009; Yanny et al. 2009). The SDSS-GSC-II catalogue contains positions, proper motions, classification, and ugriz photometry for 77 million sources down to $r \approx 20$, over 9000 square-degrees. Spagna et al. (2010) analysed 27000 FGK (sub)dwarfs in the solar neighbourhood and found evidence of a rotation-metallicity correlation, $\partial V_{\phi} / \partial[\mathrm{Fe} / \mathrm{H}] \approx 40 \div 50 \mathrm{~km} \mathrm{~s}^{-1} \mathrm{dex}^{-1}$, amongst in situ thick disk stars, located at $1 \mathrm{kpc}<|z|<3 \mathrm{kpc}$ and selected with abundance $-1.0<[\mathrm{Fe} / \mathrm{H}]<-0.5$. The thin disk contamination is expected to be negligible in this sample; however, this is not true for the halo stars, consequently, the thick disk and halo populations had to be deconvolved by assuming the superposition of two Gaussian velocity distributions.

This result was also confirmed over a wider height interval, $0.1 \mathrm{kpc}<|z|<3 \mathrm{kpc}$, by Lee et al. (2011), who analysed the kinematics of $\sim 17500$ G-type dwarfs from which they selected a subsample of bona fide thick disk stars by means of $[\mathrm{Fe} / \mathrm{H}]$ and $[\alpha / \mathrm{Fe}]$ provided by the spectroscopic SDSS sample. The fact that other studies based on the photometric SDSS sample (Ivezić et al. 2008; Bond et al. 2010; Loebman et al. 2011) did not reveal any significant rotation-metallicity correlation seems to depend on the larger errors affecting the chemical abundances derived from the ugriz passbands (Arnadottir et al. 2010; Lee et al. 2011). For these reasons, it is of particular importance to cite Kordopatis et al. (2011), who recently performed a spectroscopic survey of 700 thick disk stars using fully independent VLT/FLAMES observations and found the same correlation within the interval $1 \mathrm{kpc}<|z|<4 \mathrm{kpc}$ from the plane, where the thick disk population dominates. Finally, a positive correlation was present in the sample discussed in Gratton et al. (2003), who analysed 150 subdwarfs and early subgiants in the solar neighbourhood with homogeneous spectroscopic abundances and accurate kinematics derived from the Hipparcos catalogue.

The discussion above clearly shows that part of the burden is still on the observational side and therefore on the decisive contribution that Gaia's parallaxes will bring to this topic, along the lines discussed in Freeman \& Bland-Hawthorn (2002) and in Re Fiorentin (2012) for the MW halo.

\section{Chemical distribution}

We computed the expected gradient in $[\mathrm{Fe} / \mathrm{H}]$ along the disk of the MW at various cosmic epochs. To do that we ran a detailed chemical evolution model as described in Spitoni \& Matteucci (2011), which is an upgraded version of the original two-infall model of Chiappini et al. (1997, 2001), where details can be found. This model follows the evolution of 31 chemical species including $\mathrm{H}, \mathrm{He}, \mathrm{D}, \mathrm{C}, \mathrm{N}, \mathrm{O}, \alpha$-elements, Fe and Fe-peak elements. Stellar nucleosynthesis is taken into account in detail and includes the contributions from low- and intermediate-mass stars $\left(0.8-8 M_{\odot}\right)$, massive stars $\left(M>8 M_{\odot}\right)$ that die as supernovae (SNe) of Type II and Type Ib/c, and Type Ia SNe (white dwarfs in binary systems). One of the main assumptions of the model is that the thin disk of the Galaxy forms inside-out, namely the inner disk regions are assembled first by gas accretion of extragalactic origin (primordial chemical composition) and then the outer disk regions form on timescales that vary from 2 Gyr in the inner disk $(2 \mathrm{kpc})$ to $10 \mathrm{Gyr}$ and more at larger galactocentric distances $(<14 \mathrm{kpc})$. This ensures the formation of a gradient especially if coupled with a threshold in the gas density regulating star formation. Radial flows were not considered ${ }^{1}$.

The model adopted in this paper is Model S2IT of Spitoni \& Matteucci (2011), their Table 1: it is a two-infall model similar to that presented in Chiappini et al. (2001) and Cescutti et al. (2007). This particular model was chosen because it accurately reproduces the most recent estimate of the present time abundance gradients by Luck \& Lambert (2011) along the disk, who found $\Delta[\mathrm{Fe} / \mathrm{H}] / \Delta R=-0.062 \pm 0.002 \mathrm{dex} \mathrm{kpc}^{-1}$. Our predicted present time gradient for $\mathrm{Fe}$ is $\Delta[\mathrm{Fe} / \mathrm{H}] / \Delta R=-0.052 \mathrm{dex} \mathrm{kpc}-1$ in a galactocentric range $4-14 \mathrm{kpc}$. Given the uncertainties still existing in deriving chemical abundances, and in particular in the adoption of different model atmospheres, and considering the small difference in the galactocentric distance range adopted by us compared to Luck \& Lambert (2011), we consider our model predictions to be in reasonable agreement with the observations.

Spitoni \& Matteucci (2011) considered the gradient at $2 \mathrm{Gyr}$ since the beginning of star formation and the one at $8 \mathrm{Gyr}$. While the gradient at $8 \mathrm{Gyr}$ shows a clear increase of $[\mathrm{Fe} / \mathrm{H}]$ with decreasing galactocentric distance, the gradient at 2 Gyr shows an increase from the outer regions towards to $10 \mathrm{kpc}$, where it reaches a peak, and then a decrease from $R<10 \mathrm{kpc}$, as shown

\footnotetext{
1 Including radial flows is necessary to generate abundance gradients only if no inside-out and no threshold are assumed (Spitoni \& Matteucci 2011).
} 
A. Curir et al.: The thick disk rotation-metallicity correlation as a fossilof an "inverse chemical gradient" in the early Galaxy

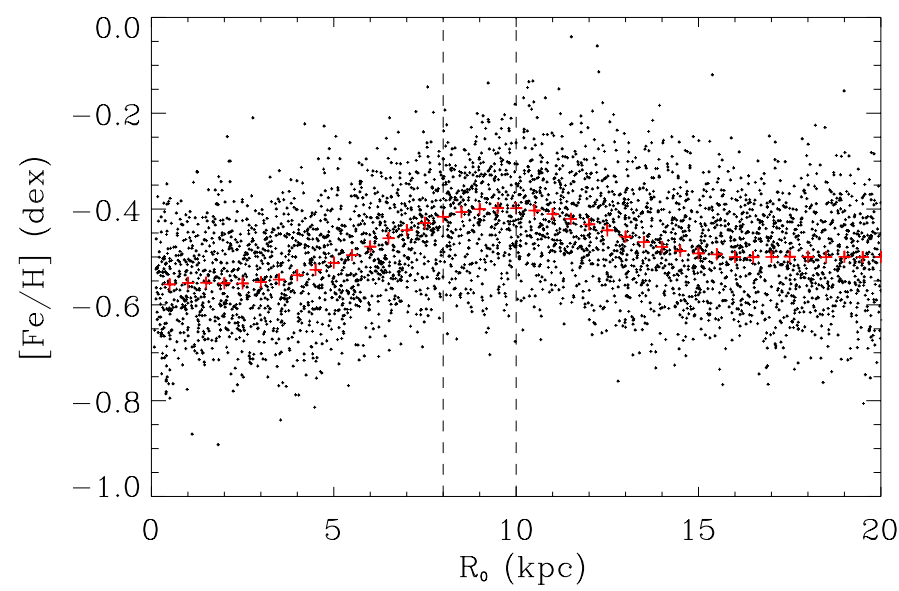

Fig. 1. Initial radial chemical distribution. The crosses show the model of Spitoni \& Matteucci (2011), while the dots, which represent the chemical distribution of the $N$-body particles at $t=0 \mathrm{Gyr}$, include an intrinsic dispersion $\sigma_{[\mathrm{Fe} / \mathrm{H}]}=0.1 \mathrm{dex}$.

in Fig. 1. This apparently strange behaviour was already present in the model of Chiappini et al. (2001) and can be explained on the basis of the inside-out disk formation, in particular, by the fact that at early epochs the efficiency of chemical enrichment in the inner regions of the disk is low due to the very large amount of infalling primordial gas. Then, at later epochs, while the star formation rate is still much higher than in the outer disk regions, the infall of primordial gas is much stronger in the outer disk. This produces the early flat gradient and the steepening of the gradient in time. We adopted a metallicity dispersion, $\sigma_{[\mathrm{Fe} / \mathrm{H}]}=0.1 \mathrm{dex}$, corresponding to the typical precision of the spectroscopic metallicity measurements for SDSS, as estimated by Allende Prieto et al. (2008).

\section{Simulations}

We used a dark matter (DM) halo containing a rotating stellar exponential disk. The DM halo has a Navarro, Frenk and White (NFW) (Navarro et al. 1997) radial density profile and structural parameters as indicated in Table 1 . These parameters are appropriate for an MW-like DM halo at redshift $z=0$.

We used MW parameters at $z=0$ to initialise our model: since we want to compare gradients observed at present time with those generated by dynamical evolution, we need to have the correct physical parameters of today. Of course, this introduces a discrepancy, because several Gyr ago the Galaxy was different. However, we believe that our model, even if it is a simple nondissipative one, is adequate to separate effects of dynamics from chemical evolution. The DM particles of the nonrotating halo have velocities given by the local equilibrium approximation (Hernquist 1993).

A stellar disk, whose mass is set to the value estimated for the MW, is embedded into our halo; the other features of the simulated disk are described in Table 2. The position of each disk particle is obtained by using the rejection method in Press et al. (1986); the disk is in gravitational equilibrium with the DM halo. We also simulated a comparison case consisting of the same disk but endowed with a bulge with a Hernquist radial density profile and mass of $1.8 \times 10^{10} M_{\odot}$ (see Table 3 ). The bulgeless disk decays in a barred configuration, while the disk with a bulge is stabilised against bar formation. We ran our two simulations using the public parallel treecode GADGET2 (Springel 2005) on the
Table 1. Halos properties (Navarro, Frenk and White profile).

\begin{tabular}{cccccccc}
\hline \hline DM & $M_{\text {vir }}$ & $R_{\text {vir }}$ & $C_{\text {vir }}$ & $R_{\max }$ & $N$ & $\epsilon$ & $M_{\mathrm{DM}}$ \\
\hline Halo & $10^{12}$ & 258 & 7.40 & 336 & $10^{7}$ & 0.11 & $1.07 \times 10^{5}$ \\
\hline
\end{tabular}

Notes. $M_{\text {vir }}$ : Halo's virial mass in $M_{\odot} ; R_{\text {vir }}$ : virial radius in kpc. $C_{\text {vir }}$ : NFW concentration parameter. $R_{\max }$ : maximum radius. $N$ : total number of Halo particles. $\epsilon$ : softening length in kpc. $M_{\mathrm{DM}}$ : mass of DM particle in $M_{\odot}$.

Table 2. Properties of the disk.

\begin{tabular}{cccccccc}
\hline \hline Stars & $M_{*}$ & $M_{\text {star }}$ & $h_{\mathrm{d}}$ & $z_{\mathrm{d}}$ & $N$ & $\epsilon$ & $Q$ \\
\hline Disk & $5.6 \times 10^{10}$ & $7.47 \times 10^{3}$ & 3.5 & 0.7 & $7.5 \times 10^{6}$ & 0.044 & 2 \\
\hline
\end{tabular}

Notes. $M_{*}$ : disk mass in $M_{\odot} . M_{\text {star }}$ : mass of the disk particle in $M_{\odot}$. $h_{\mathrm{d}}$ : disk scale length in kpc. $z_{\mathrm{d}}$ : initial disk thickness. $N$ : number of particles. $\epsilon$ : softening length in kpc. $Q$ : Toomre parameter.

Table 3. Properties of the bulge (Hernquist profile).

\begin{tabular}{lccccc}
\hline \hline Stars & $M_{*}$ & $N$ & $\epsilon$ & $a$ & $M_{\mathrm{b}}$ \\
\hline Bulge & $1.85 \times 10^{10}$ & $2.5 \times 10^{6}$ & 0.044 & 1.12 & $7.39 \times 10^{3}$ \\
\hline
\end{tabular}

Notes. $M_{*}$ : mass in $M_{\odot} . N$ : number of particles. $\epsilon$ : softening length in kpc. $a$ : Hernquist scale radius in kpc. $M_{\mathrm{b}}$ : mass of the particle in $M_{\odot}$.

cluster matrix at the CASPUR (Consorzio Interuniversitario per le Applicazioni del Supercalcolo) consortium, Rome. Both systems were left to evolve for $10 \mathrm{Gyr}$. The snapshots of the particle configurations were taken with a timestep of $0.25 \times 10^{8}$ years. To avoid the effect of relaxation of our initial conditions, we let the dynamical system to evolve for $1 \mathrm{Gyr}$ before assigning our initial metallicities ${ }^{2}$, and we analysed the redistribution of the chemical properties over the following 9 Gyr. We assigned the radial chemical distribution function defined in Sect. 3, which is shown in Fig. 1, to what we considered our initial configuration. Each particle in the configuration is tagged with a $[\mathrm{Fe} / \mathrm{H}]$ label according to this initial radial function.

\section{Results}

\subsection{Evolution of $V_{\phi}$ vs. $[\mathrm{Fe} / \mathrm{H}]$}

The evolution of the rotation-metallicity correlation $\partial V_{\phi} / \partial[\mathrm{Fe} / \mathrm{H}]$ in the barred disk model, evaluated for particles in the range $-0.55<[\mathrm{Fe} / \mathrm{H}]<-0.35$ and $V_{\phi}>50 \mathrm{~km} \mathrm{~s}^{-1}$, is shown as a dashed thick line in Figs. 3-5. This choice for the chemical and kinematical ranges allows one to consider the core of the metallicity distribution and exclude the low-velocity tail. These figures represent the distribution $V_{\phi}$ vs. [Fe/H] at different times within the solar annulus $8 \mathrm{kpc}<R_{0}<10 \mathrm{kpc}$ and for $1.5 \mathrm{kpc}<|z|<2.0 \mathrm{kpc}$. The colour-coded zones shown in the right panels of Figs. 3-5 represent the distribution of the average $\left\langle R_{0}\right\rangle$ original radii of the particles as a function of the location on the plane $\left(V_{\phi},[\mathrm{Fe} / \mathrm{H}]\right)$.

Table 4 lists the values of the correlation at various times and the time evolution of the correlation is shown in Fig. 2.

\footnotetext{
${ }^{2}$ From this point on any reference to time evolution will be given with regard to the time of injection of the chemical distribution in the simulation. Therefore $t=0$ corresponds to $1 \mathrm{Gyr}$ from the beginning of the dynamical simulation.
} 


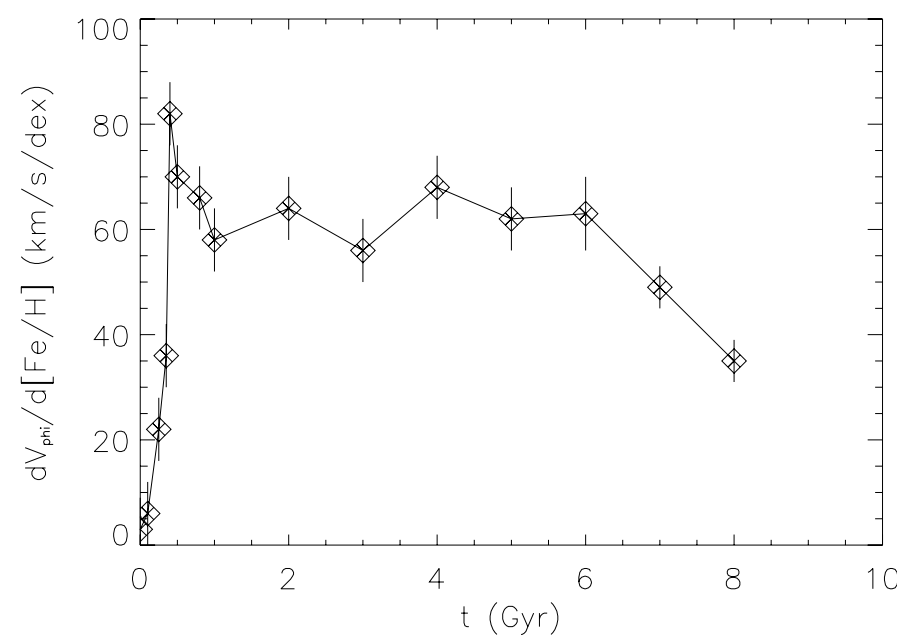

Fig. 2. Time evolution of the coefficient $\partial V_{\phi} / \partial[\mathrm{Fe} / \mathrm{H}]$.

Table 4. Evolution of the rotation-metallicity correlation.

\begin{tabular}{lcc}
\hline \hline$t$ & $n 1$ & $\partial V_{\phi} / \partial[\mathrm{Fe} / \mathrm{H}]$ \\
\hline$(\mathrm{Gyr})$ & & $\left(\mathrm{km} \mathrm{s}^{-1} \mathrm{dex}^{-1}\right)$ \\
\hline 0.0 & 29171 & $3 \pm 6$ \\
0.1 & 29135 & $6 \pm 6$ \\
0.25 & 28592 & $22 \pm 6$ \\
0.35 & 28676 & $36 \pm 6$ \\
0.4 & 28276 & $82 \pm 6$ \\
0.5 & 28926 & $70 \pm 6$ \\
0.8 & 28928 & $66 \pm 6$ \\
1.0 & 28922 & $58 \pm 6$ \\
2.0 & 32943 & $64 \pm 6$ \\
3.0 & 29324 & $56 \pm 6$ \\
4.0 & 30062 & $68 \pm 6$ \\
5.0 & 28537 & $62 \pm 6$ \\
6.0 & 31037 & $63 \pm 7$ \\
7.0 & 79295 & $49 \pm 4$ \\
8.0 & 99357 & $35 \pm 4$ \\
\hline
\end{tabular}

Notes. $t$ : time of evolution. $n 1$ : number of particles having $1.5 \mathrm{kpc}<$ $|z|<2.0 \mathrm{kpc}$.

By definition, at $t=0 \mathrm{Gyr}$ all particles belong to the annulus $8 \mathrm{kpc}<R_{0}<10 \mathrm{kpc}$ and no rotation-metallicity correlation can be present because the $[\mathrm{Fe} / \mathrm{H}]$ values are randomly drawn and assigned to the particles at that time. Because of stellar motions, at $t=0.1 \mathrm{Gyr}$ and in the $8-10 \mathrm{kpc}$ annulus, we found many particles coming from the outer and inner regions, although no rotation-metallicity correlation is present as yet.

A mild correlation appears between $t=0.2$ and $t=0.3 \mathrm{Gyr}$, which quickly increases up to $82 \pm 6 \mathrm{~km} \mathrm{~s}^{-1} \mathrm{dex}^{-1}$ at $t=0.4 \mathrm{Gyr}$ (Figs. 2 and 3). We note that this correlation is produced by the "immigration" of low-rotating and metal-poor particles from the inner regions, which populate the bottom-left corner of the right panels (as indicated by the dotted white iso-contours) and tilt the iso-density contours in the left panels. As shown in Fig. 4, the rotation-metallicity distribution is stable over several Gyr, until about 6 Gyr. As reported in Table 4, the number of particles is also nearly constant at $\approx 30000$ in the $8-10 \mathrm{kpc}$ annulus and $1.5 \mathrm{kpc}<|z|<2.0 \mathrm{kpc}$.

After $6 \mathrm{Gyr}$, we observe a significant broadening of the $V_{\phi}$ distribution due to the arrival of many particles from the inner regions of the disk that populate the slowly rotating tail of the velocity distribution $\left(V_{\phi} \lesssim 100 \mathrm{~km} \mathrm{~s}^{-1}\right)$. We note, however, that a smaller and decreasing rotation-metallicity correlation persists up to $t=8 \mathrm{Gyr}$ (cfr. Table 4). Notice also that the number of particles increases dramatically at 7 Gyr, reaching $\sim 100000$ at $t=8 \mathrm{Gyr}$, with a corresponding dramatic change in the azimuthal velocity distribution, as shown in the last two panels of Fig. 6.

This figure helps following the migration history of the particles of interest by showing in the left panels the histograms of their original radial distributions at the same evolutionary times as those used for Figs. 3-5. Clearly the peak of the distribution moves with the evolution of the disk outside of the solar annulus towards smaller initial radii.

Finally, it is worth to remark on the intriguing feature of the initial sharp peak in Fig. 2. We estimated an average timescale of $\approx 0.2$ Gy for the stars involved to oscillate around their guiding centres, a timescale compatible with the time needed for the formation of this initial spike in the evolution of the rotationmetallicity correlation. A deeper investigation on the role of the orbital features and on the dynamical orbital evolution will be the subject of a forthcoming study.

\subsection{Barred vs unbarred disks, impact of metalliticy dispersion, and impact of relaxation}

Figure 7, upper panel, shows the original radial distribution of only those particles that after 5 Gyr are found within the solar annulus ( $8 \mathrm{kpc}<R<10 \mathrm{kpc}$ ), whereas the lower panel shows the subset of these particles that have $1.5 \mathrm{kpc}<|z|<2.0 \mathrm{kpc}$. Here we can see how the outward radial migration is stronger in the barred disk: there, the bar is driving the migration since, according to Friedli et al. (1994) and Minchev \& Famey (2010), the presence of nonaxisymmetric features in the disk causes radial migration. On the other hand, it appears that a diffusion is also produced by the coarse-grained gravitational field present in our simulations, as indicated by the results of our nonbarred disk case. A fraction of this diffusion could be numerical; however, it might mimic the diffusion generated by large molecular clouds in real galaxies (Wielen 1977; Jenkins \& Binney 1990).

In the barred-disk case the bulk of the particles at $R=8-10 \mathrm{kpc}$ arrives from the inner region of the disk (Fig. 7, upper panel). Because these particles originated in internal regions affected by a stronger vertical potential, they can extend to higher heights off the plane (Fig. 7, lower panel). These results are consistent with previous studies carried out by Roškar et al. (2008) and Loebman et al. (2011).

In Fig. 8 we show the histograms describing the distribution of the differences $L_{\mathrm{fin}}-L_{\mathrm{ini}}$ between the initial and final, after 5 Gyr, angular momenta of the selected particles, in the solar annulus $8-10 \mathrm{kpc}$, for the barred and unbarred disks. In the top histograms, we present the angular momenta differences for all particles in the annulus, whereas the lower panels show the same differences for these particles with heights above the plane in the interval $1.5 \mathrm{kpc}<|z|<2 \mathrm{kpc}$. These plots show how angular momentum exchanges are more important for the barred case. The less evident skewness of the histogram for the barred case and particles at higher $|z|$ reflects the more symmetric distribution shown by the red curve in the lower panel of Fig. 7 .

To test the effect of metallicity dispersion on our results, we repeated our numerical experiment by plugging the same metallicity profile, but with different initial metallicity dispersions. As expected, the rotation-metallicity correlation is strongly dependent on the assumed initial metallicity dispersion. Indeed, at $t=5 \mathrm{Gyr}$ its value becomes $21 \pm 8 \mathrm{~km} \mathrm{~s}^{-1} \mathrm{dex}^{-1}$ if we 
A. Curir et al.: The thick disk rotation-metallicity correlation as a fossilof an "inverse chemical gradient" in the early Galaxy
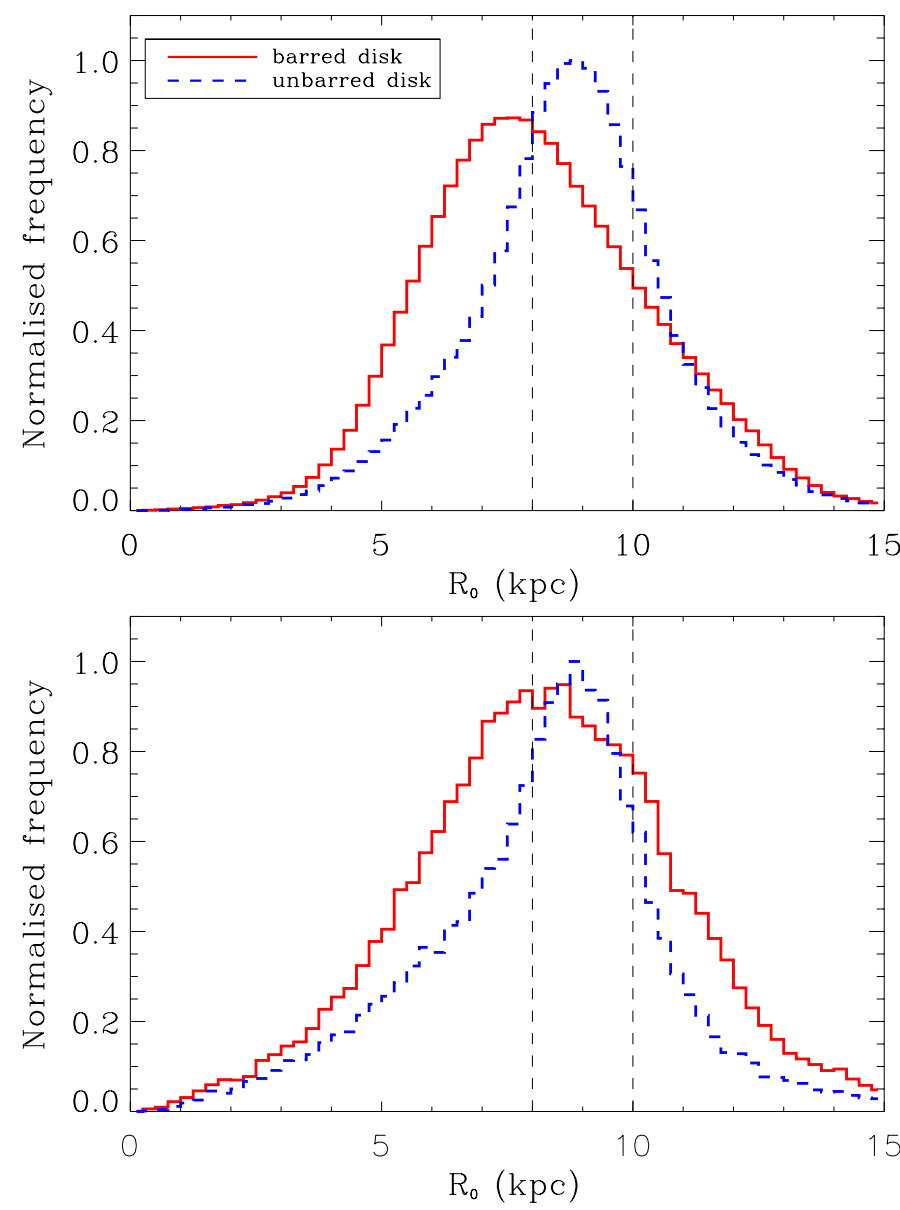

Fig. 7. Upper panel: histogram of the original radial distributions of the subset of particles found within $8 \mathrm{kpc}<R<10 \mathrm{kpc}$ after $5 \mathrm{Gyr}$. Lower panel: histogram of the original radial distributions of the same particles as in the upper panel, but with $1.5 \mathrm{kpc}<|z|<2.0 \mathrm{kpc}$.

run our model with an initial metallicity dispersion of 0.2 dex, while it becomes $157 \pm 6 \mathrm{~km} \mathrm{~s}^{-1} \mathrm{dex}^{-1}$ with an initial metallicity dispersion set to 0.05 dex.

We note that the impact on the rotation-metallicity correlation of a higher initial metallicity dispersion (a reduction in the correlation) appears to be similar to that caused by churning, which might be dominating after 6 Gyr (see Fig. 2). This will be investigated in our forthcoming study.

Finally, to address the question of the possible role of partial/incomplete dynamical relaxation, we re-assigned the initial metallicities after 2 Gyr of evolution and compared the results to the 1-Gyr case. No significant difference was found, thus confirming that our results are not the spurious effect of relaxation of the adopted initial conditions.

\subsection{Comparison to the data}

The results of Fig. 2 testify to a positive rotation-metallicity correlation that persists at $\simeq 60 \mathrm{~km} \mathrm{~s}^{-1} \mathrm{dex}^{-1}$ for several Gyr, to be compared with the $40-50 \mathrm{~km} \mathrm{~s}^{-1} \mathrm{dex}^{-1}$ measured by Spagna et al. (2010) and Lee et al. (2011).

Although we did not attempt any rigorous fit to the data, which would not be appropriate given the limitations of our galaxy model, we emphasise the consistency of the kinematics produced by our dynamical simulations with what was unveiled by the data.

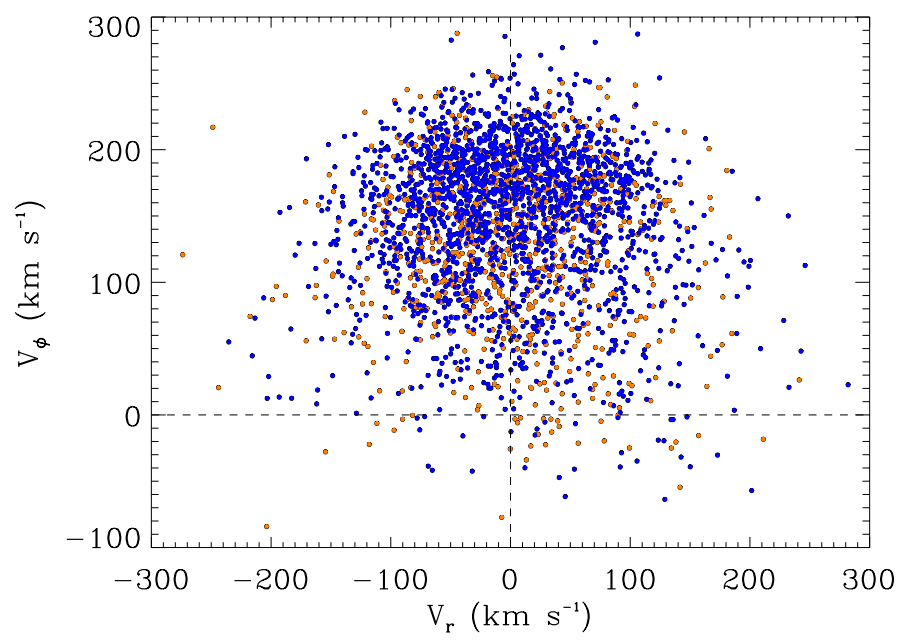

Fig. 9. Simulated distribution of the azimuthal velocity vs. radial velocity based on particles $(8 \mathrm{kpc}<R<10 \mathrm{kpc}$ and $1.5 \mathrm{kpc}<|z|<2.0 \mathrm{kpc})$ from the barred disk model at $t=5$ Gyr. Here, blue and red symbols mark the particles with simulated $[\mathrm{Fe} / \mathrm{H}]>-0.5$ and $[\mathrm{Fe} / \mathrm{H}] \leq-0.5$, respectively.

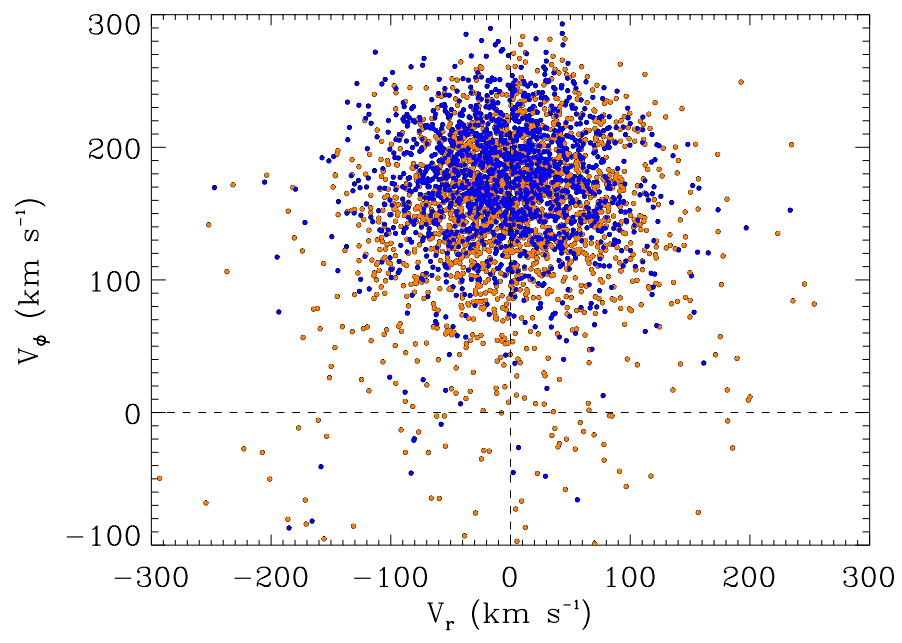

Fig. 10. Distribution of the azimuthal velocity vs. radial velocity based on 3507 stars of the SDSS-GSC-II catalog $(1.5 \mathrm{kpc}<|z|<2.0 \mathrm{kpc}$ and $-1.0 \leq[\mathrm{Fe} / \mathrm{H}]<-0.3)$. Here, blue and red symbols mark the stars with measured $[\mathrm{Fe} / \mathrm{H}]>-0.6$ and $[\mathrm{Fe} / \mathrm{H}] \leq-0.6$, respectively.

Figures 9 and 10 compare the velocity distribution, $V_{\phi}$ vs. $V_{R}$, of the $N$-body particles in the peripheral $z$-layers to the stellar distribution derived from the SDSS-GSC-II catalogue studied by Spagna et al. (2010). The theoretical distribution shows particles from the adopted barred-disk model evolved for $5 \mathrm{Gyr}$ and selected within the solar annulus at $1.5 \mathrm{kpc}<|z|<2.0 \mathrm{kpc}$. We note that, in spite of the model limitations, the two distributions appear to be quite similar, the moments of the simulated distribution $\left(\left\langle V_{\phi}\right\rangle \simeq 142 \mathrm{~km} \mathrm{~s}^{-1}, \sigma_{V \phi} \simeq 59 \mathrm{~km} \mathrm{~s}^{-1}, \sigma_{V_{R}} \simeq 70 \mathrm{~km} \mathrm{~s}^{-1}\right)$ are quite close to the values of the observed distribution $\left(\left\langle V_{\phi}\right\rangle \simeq\right.$ $159 \mathrm{~km} \mathrm{~s}^{-1}, \sigma_{V \phi} \simeq 56 \mathrm{~km} \mathrm{~s}^{-1}, \sigma_{V_{R}} \simeq 62 \mathrm{~km} \mathrm{~s}^{-1}$.

In addition, we note an excess of slowly rotating particles ( $V_{\phi} \lesssim 100 \mathrm{~km} \mathrm{~s}^{-1}$ ) with respect to the observations; these are particles coming from the innermost regions of the disk, and this excess seems to depend on the bar generated in the simulated disk, which is stronger than the MW bar and likely more efficient in displacing particles. 


\section{Discussion and conclusions}

Nondissipative mechanisms inside disks due to an irregular gravitational field as already theorised by Wielen (1977) are very efficient in promoting stellar orbital diffusion. Nonaxisymmetric structures such as spiral arms and bars promote significant radial migration (Sellwood \& Binney 2002; Minchev \& Famey 2010). Schönrich \& Binney (2009a) discussed in detail the churning and blurring processes that are responsible for the radial mixing. Through the comparison between barred and unbarred disk models, our simulations confirm the orbital diffusion in both cases but a more efficient radial mixing in the presence of a bar. These dynamical processes, which naturally produce secular migration of the stars from the disk central regions towards larger radii and higher vertical distances from the plane, represent a plausible mechanism to explain the origin of the MW thick disk.

We recall that our simulations do not include any gaseous component or star formation. We simply investigated the evolution of a system formed by collisionless particles representing the 5-10 Gyr old parent population of the thick disk component we observe today. Through this approach, we dramatically reduced the number of degrees of freedom of the model, so that we were able to investigate the details of the dynamical processes and their effects on the thick disk chemical properties in situ, i.e., at higher heights from the plane, where we assume that no significant contamination due the younger thin disk stars is present. In the light of our results, the thick disk rotation-metallicity correlation (Spagna et al. 2010; Lee et al. 2011) seems to represent an important signature of the disk evolution. This feature can indeed be explained by the role of the dynamical migration in our $N$-body simulations, once we assume an initial radial chemical gradient as that suggested by the chemical evolution models of Spitoni \& Matteucci (2011), which prescribes a positive slope for the inner early disk, $R \lesssim 10 \mathrm{kpc}$, combined with the usual decreasing slope in the outer disk. The crucial role of a positive inner slope for the primordial chemical distribution to produce a positive rotation-metallicity correlation was presented in Curir et al. (2012), where a distribution consisting of two simple linear functions with positive slope for inner radii up to $6 \mathrm{kpc}$ and a negative one outwards was plugged in the early ( $N$-body) disk ${ }^{3}$.

The scenario above is consistent with the recent results published by Cresci et al. (2010), who studied a sample of distant galaxies at redshift $z>3$ and found evidence for an inverse metallicity gradient, possibly produced by the accretion of primordial gas, which diluted the abundance of elements heavier than helium in the centre of the galaxies. Thus, as the negative correlation, $-22 \mathrm{~km} \mathrm{~s}^{-1} \mathrm{dex}^{-1}$, of the thin disk (Lee et al. 2011) results from the current - monotonically decreasing - radial gradient, the positive rotation-metallicity correlation of about 40-50 $\mathrm{km} \mathrm{s}^{-1} \mathrm{dex}^{-1}$, shown by the ancient thick disk population, can be explained by the relic signature of the inverse chemical gradient of the early ISM from which these stars formed 8-12 Gyr ago.
Acknowledgements. A.C. and P.R.F. acknowledge the financial support of the Italian Space Agency through ASI contracts I/037/08/0 and I/058/10/0 (Gaia Mission - The Italian Participation to DPAC). A.C. and G.M. acknowledge the financial support of INAF through the PRIN 2010 grant No. 1.06.12.02: "Towards an Italian Network for Computational Cosmology". The simulations were carried out at CASPUR, with CPU time assigned through the Standard HPC grant 2010: "Secular processes in disk galaxies and the formation of the thick disk". We thank Alvaro Villalobos for his contribution in providing the initial condistions of the numerical simulations. Thanks are due to the referee Ralph Schönrich for several comments, which helped us improve the manuscript.

\section{References}

Abadi, M. G., Navarro, J. F., Steinmetz, M., \& Eke, V. R. 2003, ApJ, 597, 21 Abazajian, K. N., Adelman-McCarthy, J. K., Agüeros, M. A., et al. 2009, ApJS, 182,543

Allende Prieto, C., Thirupathi, S., Beers, T., et al. 2008, AJ, 136, 2070

Arnadottir, A. S., Feltzing, S., Lundström, I., et al. 2010, A\&A, 521, 40

Bond, N. A., Ivezić, Ž., Sesar, B., et al. 2010, ApJ, 716, 1

Bournaud, F., \& Elmegreen, B. 2009, ApJ, 694, L158

Brook, C. B., Gibson, B. K., Martel, H., \& Kawata, D. 2005, ApJ, 630, 298

Cescutti, G., Matteucci, F., François, P., Chiappini, C. 2007, A\&A, 462, 943

Chiappini, C. 2009, IAU Symp., 254, 191

Chiappini, C., Matteucci, F., \& Gratton, R. 1997, ApJ, 477, 765

Chiappini, C., Matteucci, F., \& Romano, D. 2001, ApJ, 554, 1044

Cresci, G., Mannucci, F., Maiolino, R., et al. 2010, Nature, 467, 811

Curir, A., Spagna, A., Lattanzi, M. G., Murante, G., \& Re Fiorentin, P. 2012, in

Assembling the Puzzle of the Milky Way, EpJ Web Conf., 19, 10003

Freeman, K. C. 1987, ARA\&A, 25, 603

Freeman, K., \& Bland-Hawthorn, J. 2002, ARA\&A, 40, 487

Friedli, D., Benz, W., \& Kennicutt, R. 1994, ApJ, 430, L105

Gratton, R. G., Carretta, E., Desidera, S., et al. 2003, A\&A, 406, 131

Haywood, M. 2008, MNRAS, 388, 1175

Hernquist, L. 1993, ApJS, 86, 389

Ivezić, Ž., Sesar, B., Jurić, M., et al. 2008, ApJ, 684, 287

Jenkins, A., \& Binney, J. 1990, MNRAS, 245, 305

Kordopatis, G., Recio-Blanco, A., de Laverny, P., et al. 2011, A\&A, 535, A107

Lasker, B. M., Lattanzi, M. G., McLean, B. J., et al. 2008, AJ, 136, 735

Lee, Y. S., Beers, T. C., An, D., et al. 2011, ApJ, 738, 187

Loebman, S., Roškar, R., Debattista, V. P., et al. 2011, ApJ, 737, 8

Luck, R. E., \& Lambert, D. L. 2011, ApJ, 142, 136L

Martel, H., Richard, S., Brook, C. B., et al. 2011, in IAU Symp., 277, in press

Matteucci, F., \& Francois, P. 1989, MNRAS, 239, 885

Minchev, I., \& Famaey, B. 2010, ApJ, 722, 112

Morrison, H. L., Helmi, A., Sun, J., et al. 2009, ApJ, 694, 130

Navarro, J. F., Frenk, C. S., \& White, S. D. M. 1997, ApJ, 190, 493

Press, W. H., Flannery, B. P., Teukolsky, S. A., \& Vetterling, W. T. 1986, Numerical Recipes (Cambridge: University Press)

Quinn, P. J., Hernquist, L., \& Fullagar, D. P. 1993, ApJ, 403, 74

Qu, Y., Di Matteo, P., Lehnert, M. D., \& van Driel, W. 2011, A\&A, 530, A10

Re Fiorentin, P. 2012, in The Chemical Evolution of the Milky Way, Sexten Center for Astrophysics (SCfA), Sesto Pusteria, Bolzano, Italy, January 23-27

Robin, A. C., Haywood, M., Creze, M., Ojha, D. K., \& Bienayme, O. 1996, A\&A, 305, 125

Roškar, R., Debattista, V. P., Stinson, G. S., et al. 2008, ApJ, 675, L65

Schönrich, R., \& Binney J. 2009a, MNRAS, 396, 203

Schönrich, R., \& Binney J. 2009b, MNRAS, 399, 1145

Sellwood, J. A., \& Binney, J. J. 2002, MNRAS, 336, 785

Spagna, A., Lattanzi, M. G., Re Fiorentin, P., \& Smart, R. L. 2010, A\&A, 510, L4

Spitoni, E., \& Matteucci, F. 2011, A\&A, 531, A72

Springel, V. 2005, MNRAS, 364, 1105

Villalobos, Á., \& Helmi, A. 2008, MNRAS, 391, 1806

Wielen, R. 1977, A\&A, 60, 263

Yanny, B., Rockosi, C., Newberg, H. J., et al. 2009, AJ, 137, 4377

Pages 7 to 11 are available in the electronic edition of the journal at http://www . aanda. org

\footnotetext{
3 This proves that the radial location of the peak of the metallicity distribution adopted in this paper has no relevance for the formation of the rotation-metallicity correlation.
} 
A. Curir et al.: The thick disk rotation-metallicity correlation as a fossilof an "inverse chemical gradient" in the early Galaxy
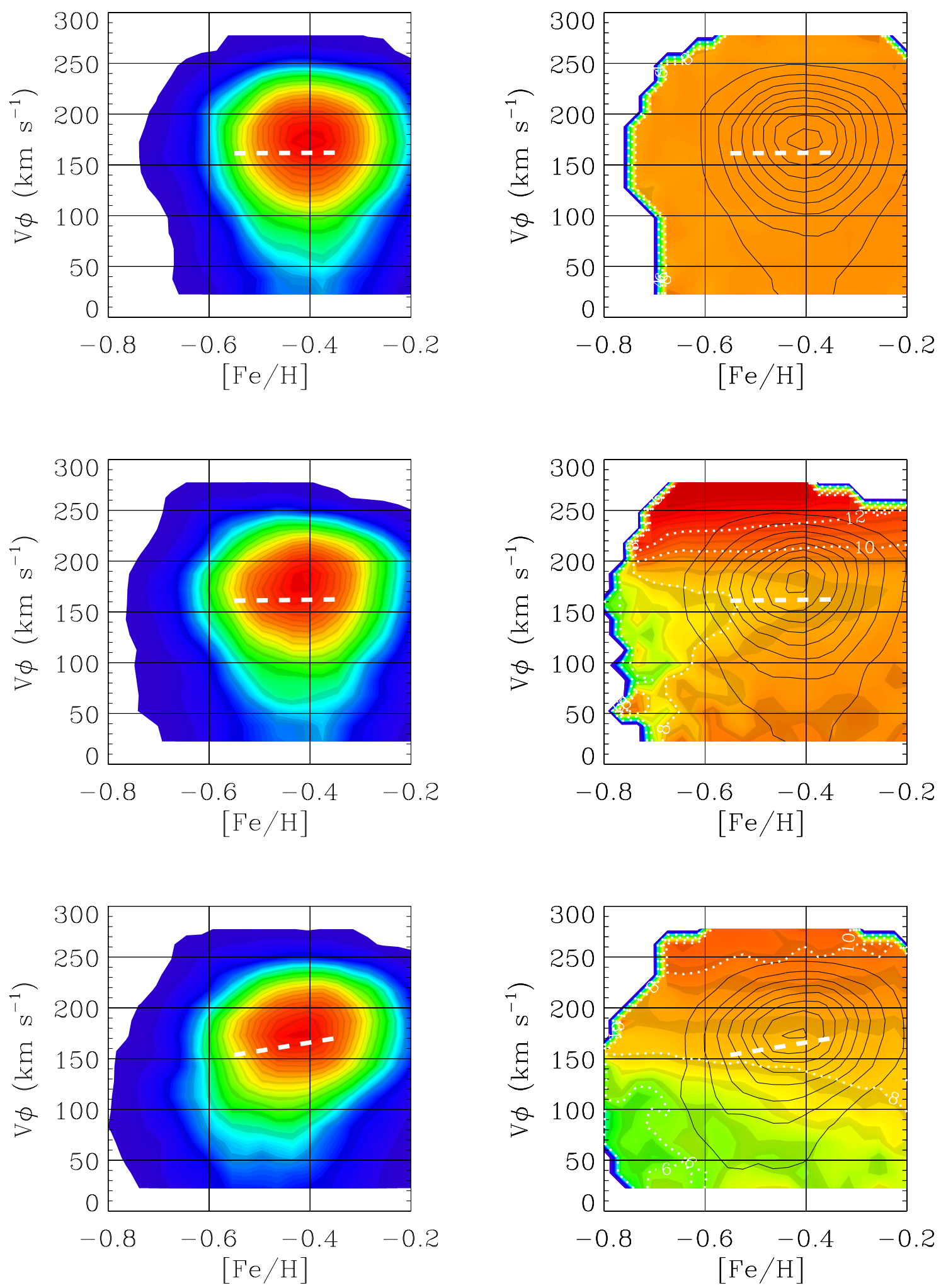

Fig. 3. Barred-disk model. Distribution of the azimuthal velocity and metallicity after an evolution of $0,0.1,0.4$ Gyr. Left panels: colour contour plot showing the rotation-metallicity distribution, $V_{\phi}$ vs. $[\mathrm{Fe} / \mathrm{H}]$, of the particles in the solar annulus, $8 \mathrm{kpc}<R<10 \mathrm{kpc}$ and $1.5 \mathrm{kpc}<|z|<2.0 \mathrm{kpc}$. The dashed white line indicates the linear fit in the central region of the distribution, including all particles with $-0.55<[\mathrm{Fe} / \mathrm{H}]<-0.35$ and $V_{\phi}>50 \mathrm{~km} \mathrm{~s}^{-1}$. Right panels: the colour contour plot represents the distribution of the mean original radius, $\left\langle R_{0}\right\rangle$, as a function of $\left([\mathrm{Fe} / \mathrm{H}], V_{\phi}\right)$ of the same particles shown in the left plot. Here, the dotted white lines mark the iso-contours at $\left\langle R_{0}\right\rangle=6 \mathrm{kpc}, 8 \mathrm{kpc}, 10 \mathrm{kpc}$, and $12 \mathrm{kpc}$. The black contours indicate the iso-density levels of the $\left([\mathrm{Fe} / \mathrm{H}], V_{\phi}\right)$ distribution shown in the left panel. 

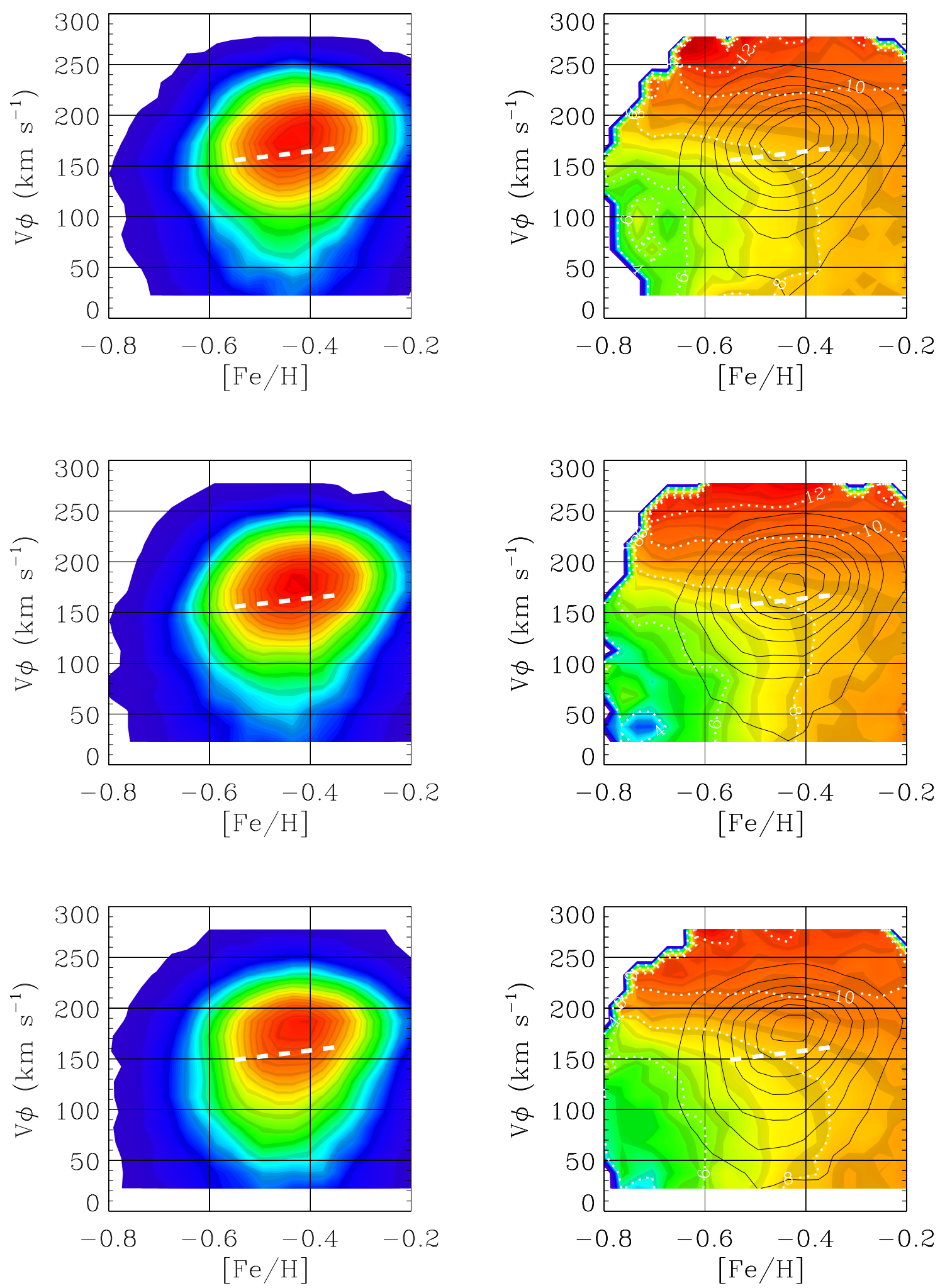

Fig. 4. Distribution of the azimuthal velocity and metallicity after an evolution of 1, 3, 5 Gyr. 
A. Curir et al.: The thick disk rotation-metallicity correlation as a fossilof an "inverse chemical gradient" in the early Galaxy
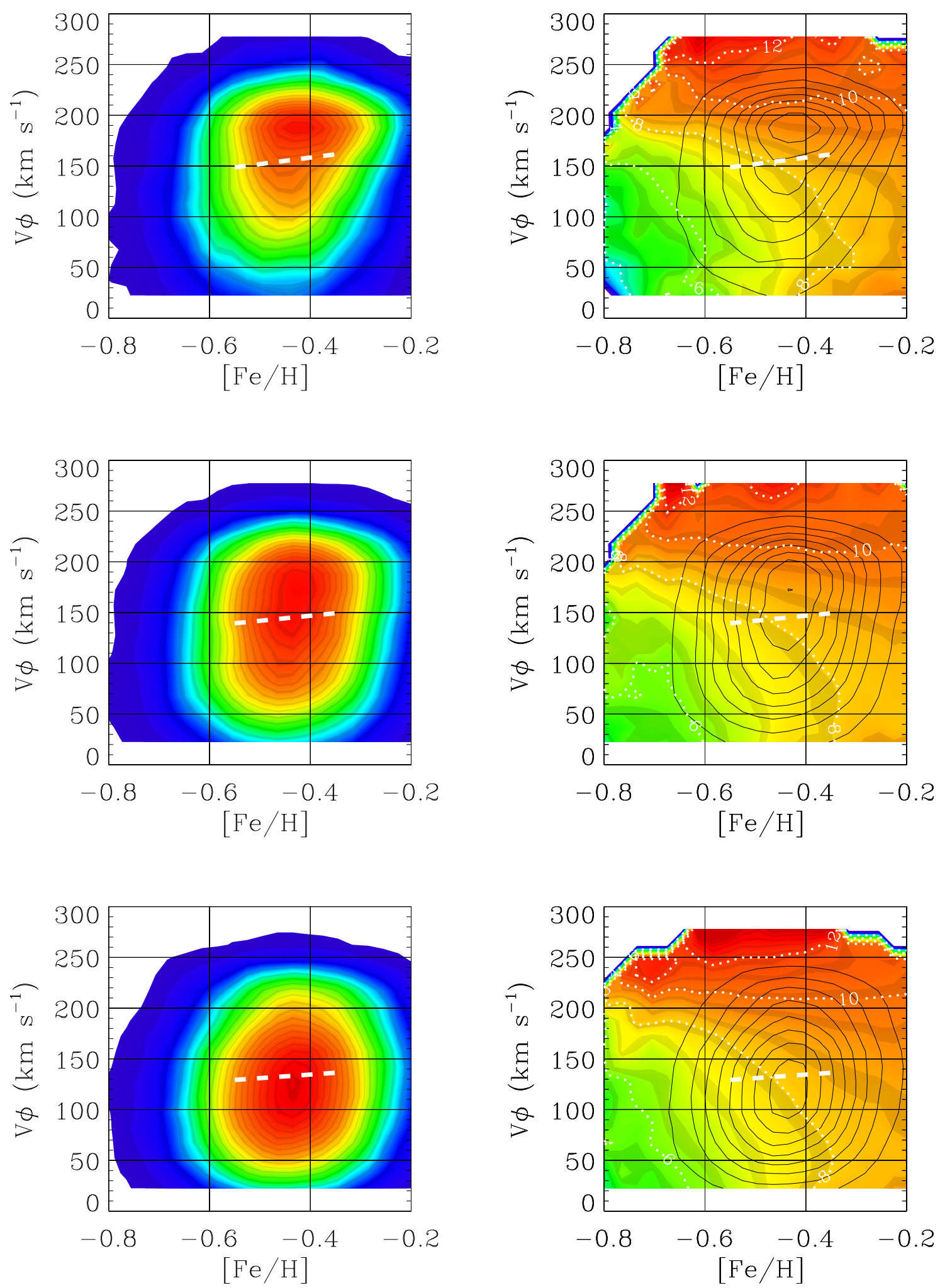

Fig. 5. Distribution of the azimuthal velocity and metallicity after an evolution of 6, 7, 8 Gyr. 

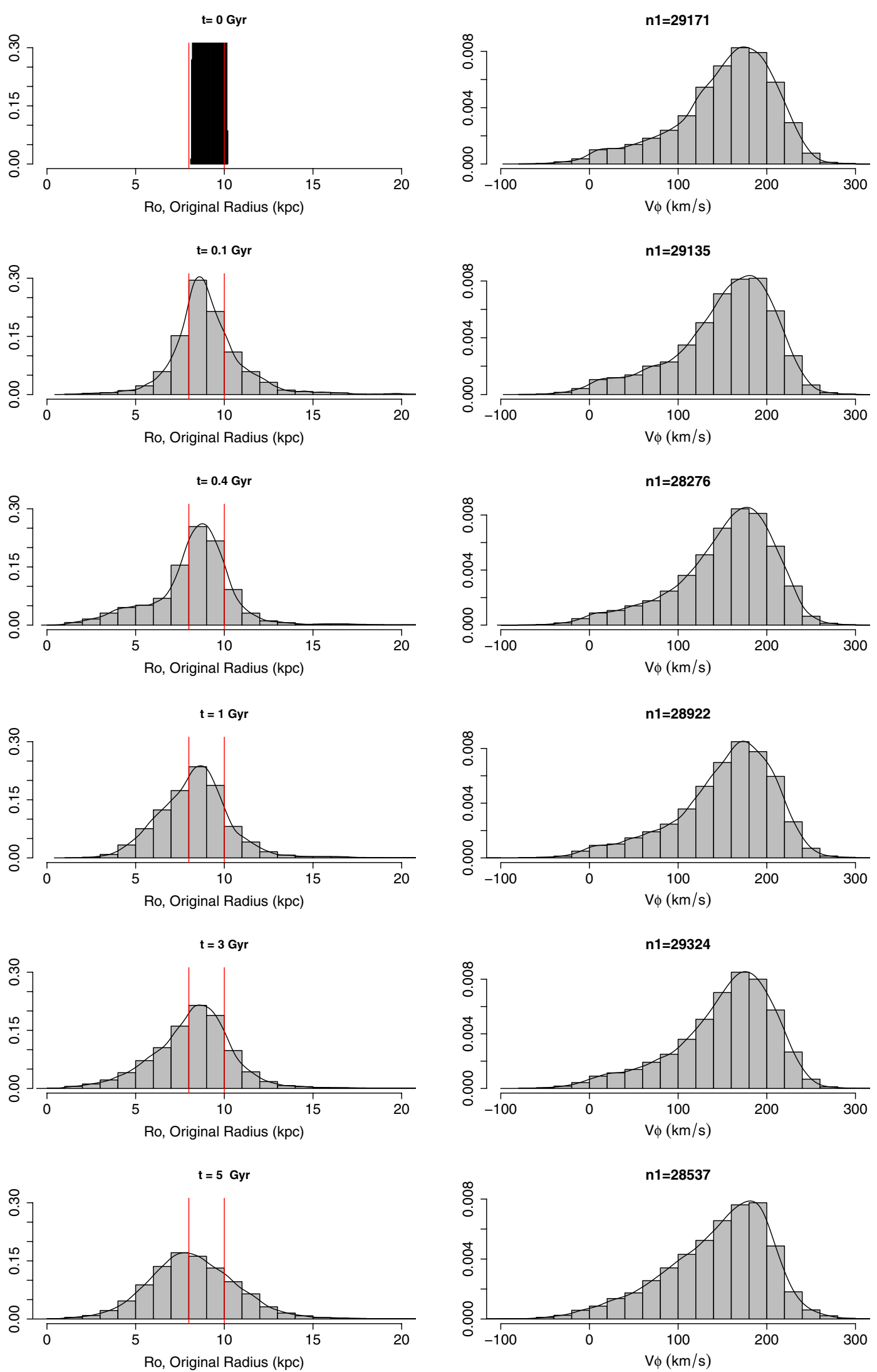

Fig. 6. Left panels: normalised histograms of the original radii of the subset of particles found within $8 \mathrm{kpc}<R<10 \mathrm{kpc}$ that have $1.5 \mathrm{kpc}<|z|<$ $2 \mathrm{kpc}$ at different evolutionary times. Right panels: normalised distributions of the azimuthal velocity at the same times. 
A. Curir et al.: The thick disk rotation-metallicity correlation as a fossilof an "inverse chemical gradient" in the early Galaxy
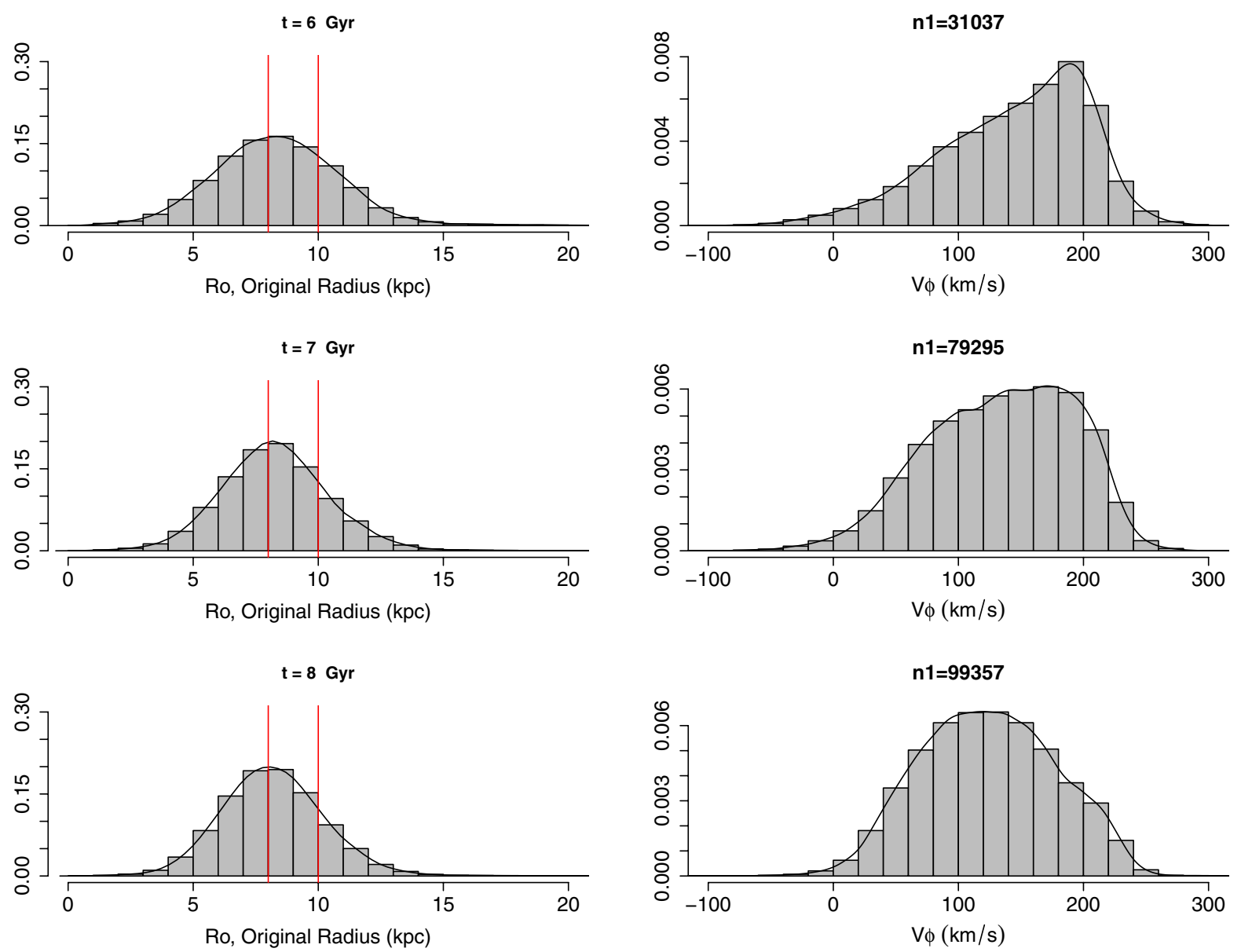

Fig. 6. continued.

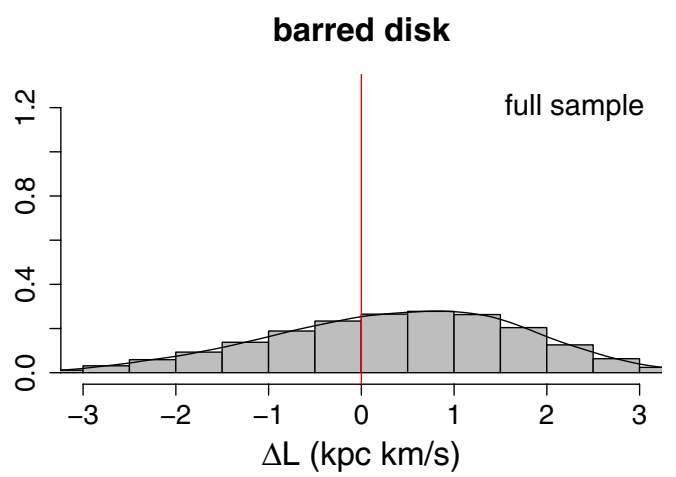

unbarred disk
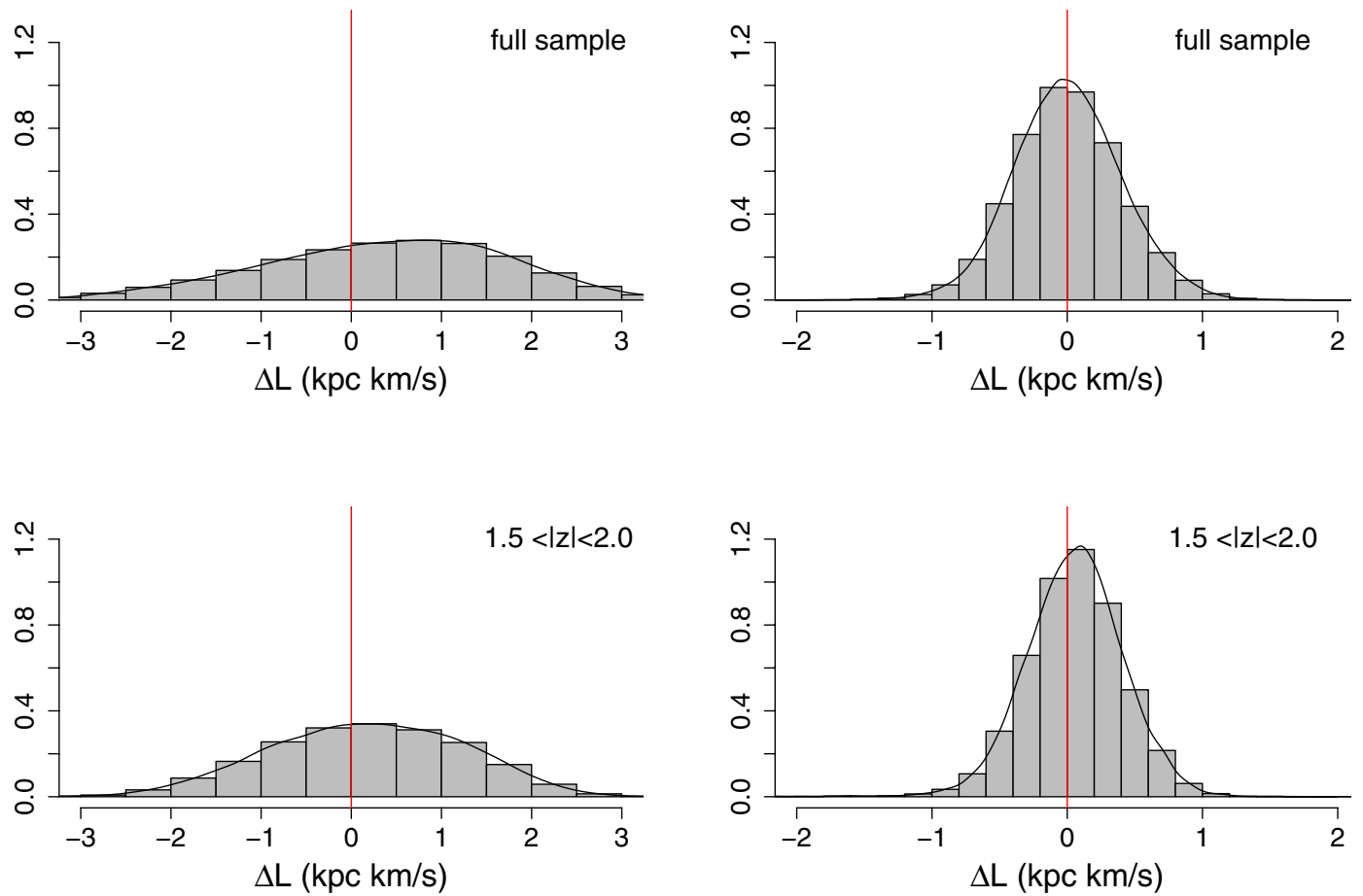

Fig. 8. Normalised distributions of the differences $L_{\mathrm{fin}}-L_{\text {ini }}$ between initial and final total angular momenta (after 5 Gyr) of the particles inside the solar annulus for the barred and unbarred disk (the curves represent an interpolation of the histogram). 